\title{
Spectroscopic study of the Carbon-Enhanced Metal-Poor star: HE 0110-0406
}

\author{
Meenakshi Purandardas ${ }^{1,2}$, Aruna Goswami ${ }^{1}$, V. H. Doddamani ${ }^{2}$ \\ ${ }^{1}$ Indian Institute of Astrophysics, Koramangala, II block, Bangalore, Karnataka, 560034, India. \\ ${ }^{2}$ Department of Physics, Bangalore University, Jnana Bharathi Campus, Karnataka, 560056, India.
}

\begin{abstract}
The halo system of the Milky way comprises the vast majority of the presently observed metal-poor $([\mathrm{Fe} / \mathrm{H}]<-1.0)$ and very metal-poor $([\mathrm{Fe} / \mathrm{H}]<-2.0)$ stars. A sizable fraction $(\sim 30 \%)$ of the metal-poor stars with carbon enhancement $([\mathrm{C} / \mathrm{Fe}] \geq 0.7)$ are called the Carbon-Enhanced Metal-Poor (CEMP) stars. Studies on these objects are of special interest, as they bear the fossil records of nucleosynthesis of the earliest generation of stars. Thus they provide insight into the nucleosynthesis and chemical evolution of the early universe. In the recent past, although these stars have drawn considerable attention as far as their spectroscopic studies are concerned, several questions still remain poorly understood such as the origin, production mechanism(s) and distribution of carbon and neutron-capture elements abundances exhibited by these objects. A comprehensive study of the abundance patterns of various heavy elements in these objects can give important clues to these questions. We have undertaken to perform chemical composition studies of a selected sample of CEMP stars using Hanle Echelle Spectrograph on the 2-m Himalayan Chandra Telescope. In this work, we present abundance results for HE 0110-0406. Our analysis shows this object to exhibit characteristic properties of $\mathrm{CH}$ giants. The abundance distribution patterns and the elemental abundance ratios are critically examined based on existing theories to understand their origin and evolution.
\end{abstract}

Keywords: Metal-poor stars - Abundances - chemically peculiar - nucleosynthesis

\section{Introduction}

Carbon stars are first realized by Angelo Secchi (Secchi 1868) as a distinct group of stars that are characterised by dominant features due to carbon molecular bands such as $\mathrm{C}_{2}, \mathrm{CH}$ and $\mathrm{CN}$. In the atmosphere of carbon stars, the carbon to oxygen $(\mathrm{C} / \mathrm{O})$ is $>1$. Bidelman (1956) introduced $\mathrm{CH}$ stars as a special group of carbon stars that show strong $\mathrm{CH}$ band in their spectra. The fraction of carbonenhanced objects is significantly large at low metallicity (Beers et al. 1992, Christlieb 2003) and the number increases with decreasing metallicity (Beers \& Christlieb 2005, and references therein). The Carbon-Enhanced Metal-Poor (CEMP) stars enriched with s-process elements are very metal-poor counterparts of $\mathrm{CH}$ stars and both belong to the same group (Masseron 2010).

Initial studies on high resolution spectroscopic analysis of a few stars from HK survey by Barbuy et al. (1997) and Norris et al. (1997) show that these stars exhibit enhancement of carbon and s-process elements. A CEMP star, CS 22892-052 identified by HK survey is found enhanced in rprocess elements (Sneden et al. 1996). The discovery of enhancement of neutron capture elements 
in a large fraction of CEMP stars promoted many spectroscopic studies to explore the origin of this abundance anomalies. However, the origin of the enhancement of carbon and neutron-capture elements exhibited by these objects are not clearly understood. The widely accepted scenario to explain the observed enhancement of s-process elements is the mass transfer from a binary companion. According to this, the currently observed CEMP star is the less massive companion in a binary system and the more massive primary companion had already passed through an AGB phase and died as a white dwarf (McClure 1983, 1984). The primary lost all its masses while evolving through the AGB phase and transferred the material synthesized through mass transfer mechanisms. Roche-lobe overflow is thought to be responsible for the mass transfer between close binary systems, and wind assisted Roche-lobe overflow is believed to dominate at intermediate separation between the binaries. Mass transfer by wind may occur in wider binaries (Arentsen 2018). The radial velocity variations exhibited by CH stars and CEMP stars (McClure (1983,1984), Preston \& Sneden (2001), Lucatello et al. (2005)) support the binary mass transfer scenario as the possible reason for the observed enhancement. However, precision radial velocity monitoring of a sample of twenty two CEMP stars by Hansen et al. (2016a, b) shows that about $24 \%$ of the stars are either single or with extremely large period. If single, the origin of the enhancement in $\mathrm{C}$ and neutron-capture elements could be intrinsic. This would however require further theoretical studies for which detailed chemical composition studies of an extended sample could provide useful constraints. We have undertaken to perform a detailed chemical composition studies for a large sample of potential $\mathrm{CH}$ star candidates from Goswami (2005) and Goswami et al. (2007, 2010). In this work we present elemental abundance results for the object HE 0110-0406. The basic data for this object is presented in Table 1.

In Section 2, we describe the methodology and in Section 3 we have presented abundance analysis. Interpretation of the abundance results are presented in Section 4. Conclusions are presented in Section 5.

\section{Methodology}

\subsection{Observations and Data Reduction}

The high resolution spectra of HE 0110-0406 were obtained with the high resolution $(\lambda / \delta \lambda \sim$ 60,000) fiber fed Hanle Echelle SPectrograph (HESP) attached to the 2-m Himalayan Chandra Telescope (HCT), at the Indian Astronomical Observatory, Hanle. We have acquired four spectra, each of 45 minutes exposure time for this object. The spectra are then combined, the resulting spectrum has $\mathrm{S} / \mathrm{N}>60$, and suitable for abundance analysis. Sample spectra is shown in Figure 1.

Data reduction is performed using Image Reduction and Analysis Facility (IRAF) software. The procedure involves trimming, bias subtraction, flat correction, wavelength calibration and dispersion correction and continuum fitting. The normalized continuum fitted spectrum is used for spectral analysis.

\subsection{Radial velocity}

The radial velocity of the star is determined by using a large number of clean unblended lines on the spectrum. The estimated radial velocity of the star is found to be $-44.4 \pm 3.8 \mathrm{Kms}^{-1}$.

\subsection{Determination of stellar atmospheric parameters}

Absorption lines due to neutral and ionized elements are identified by over-plotting the spectrum of Arcturus on the spectrum of HE 0110-0406. The equivalent widths of clean spectral lines are 

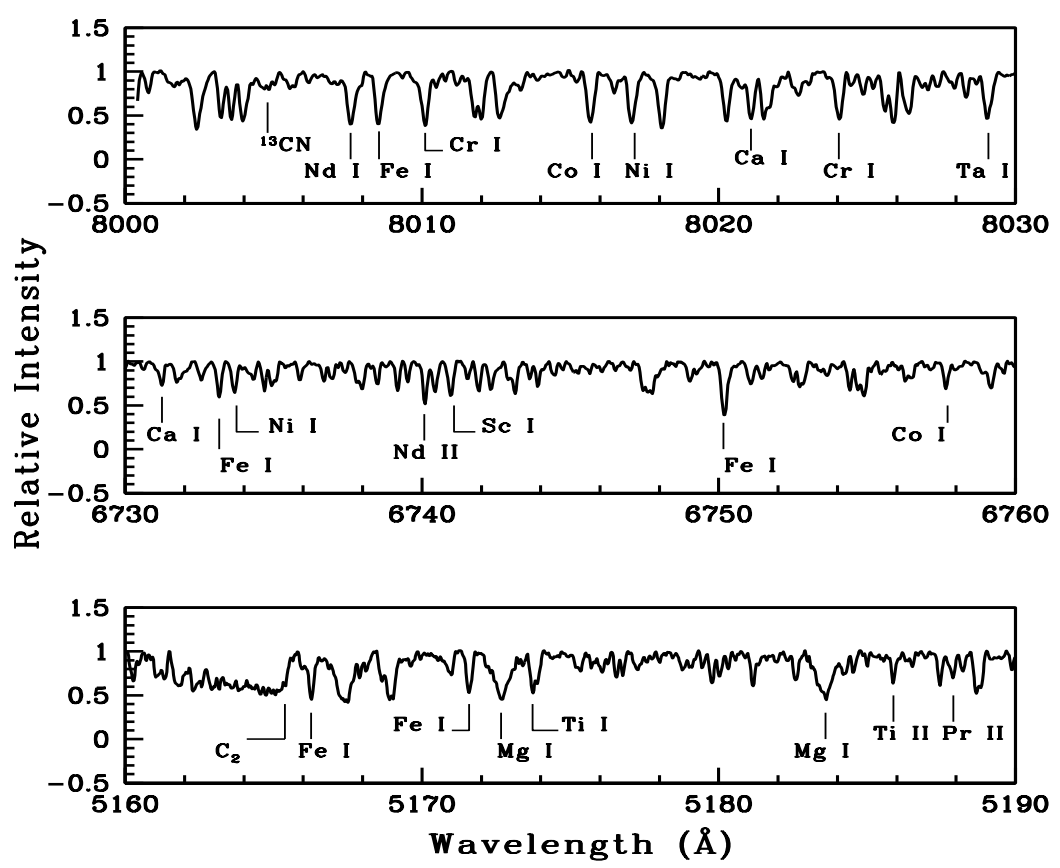

Figure 1: Sample spectra.

measured. The lines are selected to have the excitation potential in the range $0.0-5.0 \mathrm{eV}$ and the equivalent widths in the range $20-180 \mathrm{~m} \AA$. A master line list including all the clean unblended lines due to the elements are prepared. The $\log g$ and excitation potential of the lines are selected from Kurucz database. The equivalent widths of a set of Fe I and Fe II lines are used for the estimation of atmospheric parameters based on a standard Local Thermodynamic Equilibrium (LTE) analysis using model atmospheres. Model atmospheres are selected from the Kurucz grid of model atmospheres with no convective overshooting (http://cfaku5.cfa.hardvard.edu/).

A trend between the iron abundance obtained from the Fe I lines and the excitation potential of these lines with a zero slope determines the effective temperature. The abundance obtained from the Fe I lines that do not show any dependence on the reduced equivalent width $\left(W_{\lambda} / \lambda\right)$ defines the microturbulent velocity. The surface gravity $\log g$ is taken to be that value for which the abundances obtained from the Fe I and Fe II lines are nearly the same. Abundance of various elements are determined from the corresponding equivalent widths of the lines using recent version of MOOG of Sneden (1973) and the adopted model atmosphere. The derived atmospheric parameters for HE $0110-0406$ is presented in Table 2.

Table 1: Basic data for HE 0110-0406

\begin{tabular}{lccccccccc}
\hline Star & RA(2000) & Dec. $(2000)$ & B & V & J & H & K & $\begin{array}{l}\text { Exposure } \\
\text { (seconds) }\end{array}$ & Date of obs. \\
\hline HE 0110-0406 & 011237.16 & -035029.15 & 13.77 & 12.48 & 10.52 & 9.98 & 9.86 & $2700(4)^{a}$ & $24-12-2017$ \\
\hline
\end{tabular}

${ }^{a}$ Number inside the parenthesis is the number of frames taken.

\section{Abundance analysis}

We have performed a detailed abundance analysis of HE 0110-0406 based on high resolution spectroscopy. We could estimate the abundance of 22 elements which include the light elements carbon 
Table 2: Derived atmospheric parameters for HE 0110-0406.

\begin{tabular}{lccccccc}
\hline Star & $\begin{array}{c}\mathrm{T}_{\text {eff }} \\
(\mathrm{K})\end{array}$ & $\begin{array}{c}\log \mathrm{g} \\
\mathrm{cgs}\end{array}$ & $\begin{array}{c}\zeta \\
\left(\mathrm{km} \mathrm{s}^{-1}\right)\end{array}$ & {$[\mathrm{Fe} \mathrm{I} / \mathrm{H}]$} & {$[\mathrm{Fe} \mathrm{II} / \mathrm{H}]$} & $\begin{array}{c}\mathrm{V}_{r}{ }^{a} \\
\left(\mathrm{~km} \mathrm{~s}^{-1}\right)\end{array}$ & $\begin{array}{c}\mathrm{V}_{r}{ }^{b} \\
\left(\mathrm{~km} \mathrm{~s}^{-1}\right)\end{array}$ \\
\hline HE 0110-0406 & 4670 & 1.00 & 1.92 & $-1.31 \pm 0.09$ & $-1.29 \pm 0.12$ & $-44.40 \pm 3.8$ & $-32.85 \pm 2.3$ \\
\hline
\end{tabular}

and nitrogen, odd-z elements $\mathrm{Na}, \alpha$ - and Fe-peak elements $\mathrm{Mg}, \mathrm{Ca}, \mathrm{Sc}, \mathrm{Ti}, \mathrm{V}, \mathrm{Cr}, \mathrm{Mn}, \mathrm{Co}, \mathrm{Ni}$ and $\mathrm{Zn}$. We have also estimated the abundance of neutron-capture elements $\mathrm{Sr}, \mathrm{Y}, \mathrm{Ba}, \mathrm{La}, \mathrm{Ce}, \mathrm{Pr}, \mathrm{Nd}, \mathrm{Sm}$, and $\mathrm{Eu}$. The abundance analysis results are presented in Table 3.

\subsection{Carbon and Nitrogen}

Abundance of carbon is estimated from the spectrum synthesis calculation of $\mathrm{C}_{2}$ band at $5165 \AA$. The molecular lines used for the synthesis is taken from the Kurucz database. Carbon is found to be enhanced with $[\mathrm{C} / \mathrm{Fe}] \approx 0.73$. The observed spectrum is fitted with the synthetic spectrum and shown in Figure 2. The carbon isotopic ratio ${ }^{12} \mathrm{C} /{ }^{13} \mathrm{C}$ is determined from the spectrum synthesis calculation of the $\mathrm{CN}$ band at $8005 \AA .{ }^{12} \mathrm{C} /{ }^{13} \mathrm{C}$ is found to be $\sim 45$. The nitrogen abundance is determined from the spectrum synthesis of $\mathrm{CN}$ band at $4215 \AA$. Nitrogen is moderately enhanced $([\mathrm{N} / \mathrm{Fe}] \approx 0.63)$.

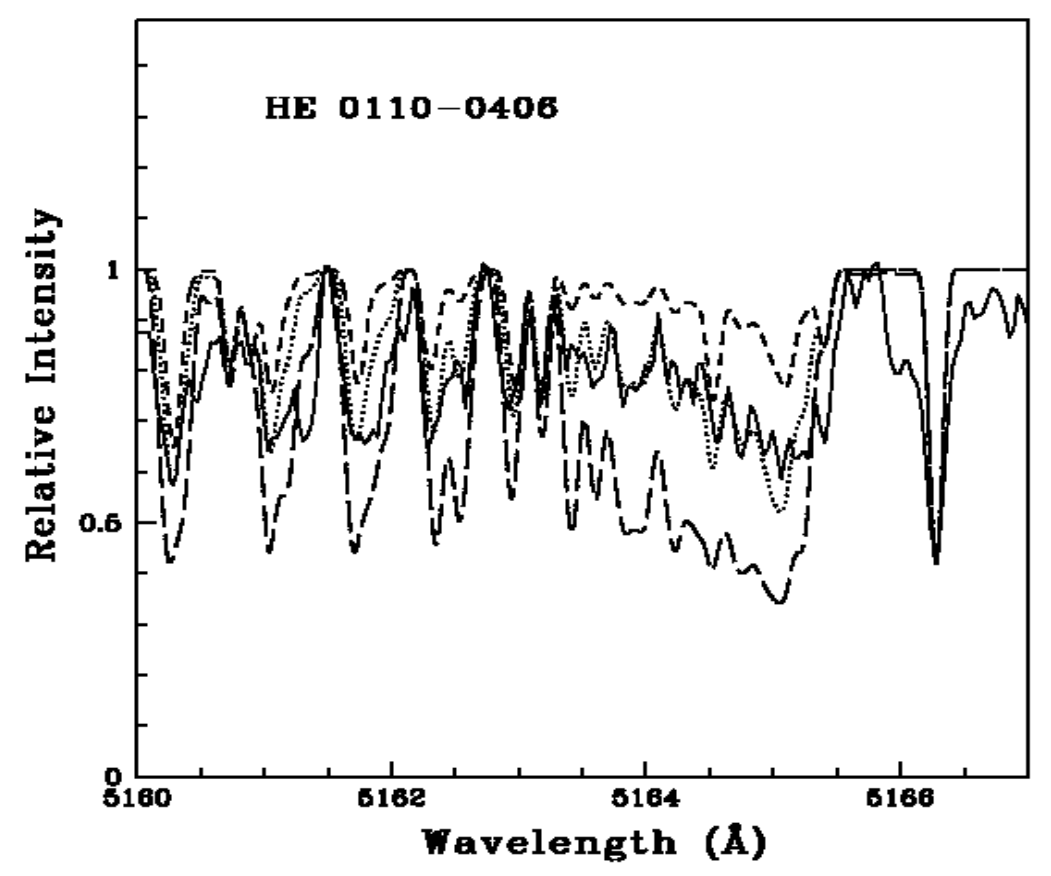

Figure 2: Synthesis of $\mathrm{C}_{2}$ band around $5165 \AA$. Dotted line represents synthesized spectra and the solid line indicates the observed spectra. Short dashed line represents the synthetic spectra corresponding to $\Delta[\mathrm{C} / \mathrm{Fe}]=-0.3$ and long dashed line corresponds to $\Delta[\mathrm{C} / \mathrm{Fe}]=+0.3$

\subsection{Na, Mg, Ca, Sc, Ti, V}

The sodium abundance is estimated from a single line at $5682.63 \AA$ which gives a value of $[\mathrm{Na} / \mathrm{Fe}] \approx$ 0.60 . HE $0110-0406$ exhibits mild enhancement of $\mathrm{Mg}$ with $[\mathrm{Mg} / \mathrm{Fe}] \approx 0.27$ which is estimated from a single line at $5711.09 \AA$. Abundances of $\mathrm{Ca}$ and $\mathrm{Ti}$ are found to be near solar. Spectrum synthesis of 
Sc II at $6245.63 \AA$ returns a value with $[\mathrm{Sc} / \mathrm{Fe}] \approx-0.14$. Vanadium abundance is estimated from the spectrum synthesis calculation of the line at $4864.731 \AA$; Vanadium is under abundant with [V/Fe] $\approx$ -0.45. Hyperfine structures for Sc and V are taken from Prochaska \& McWilliam (2000).

\subsection{Cr, Co, Mn, Ni, Zn}

The chromium and cobalt abundances derived using equivalent width measurements of a few unblended good lines of $\mathrm{Cr}$ and $\mathrm{Co}$ give near solar values for these two elements. Spectrum synthesis calculation of Mn I line at $6013.51 \AA$ is used for the abundance determination with the hyperfine structure taken from Prochaska \& McWilliam (2000). Mn shows mild enhancement with [Mn/Fe] $\approx 0.18$. Ni abundance is estimated from a single line at $6643.63 \AA$. Ni is moderately enhanced with $[\mathrm{Ni} / \mathrm{Fe}] \approx 0.45 . \mathrm{Zn}$ is found to be under abundant $([\mathrm{Zn} / \mathrm{Fe}] \approx-0.41)$.

\subsection{Sr, Y, Zr}

The light s-process elements $\mathrm{Sr}$ and $\mathrm{Y}$ are found to be enhanced with $[\mathrm{Sr} / \mathrm{Fe}] \approx 0.78$ and $[\mathrm{Y} / \mathrm{Fe}] \approx$ 1.28. The abundance of $\mathrm{Zr}$ could not be estimated.

\subsection{Ba, La, Ce, Pr, Nd, Sm, Eu}

Heavy s-process elements such as $\mathrm{Ba}, \mathrm{La}, \mathrm{Ce}, \mathrm{Nd}$ and $\mathrm{Sm}$ are found to be enhanced. We could determine only the upper limit for $\mathrm{Ba}$. The hyperfine structure for the spectrum synthesis calculation of $\mathrm{Ba}$ is taken from McWilliam (1998). The r-process elements $\mathrm{Pr}$ and Eu are enhanced with values $[\mathrm{Pr} / \mathrm{Fe}] \sim 1.39$ and $[\mathrm{Eu} / \mathrm{Fe}] \sim 0.52$ respectively.

\section{Interpretation of our results}

Our object HE 0110-0406 is found to be a $\mathrm{CH}$ giant with enhanced carbon and moderately enhanced nitrogen. The estimated carbon isotopic ratio for this object $\sim 45$, is also reported for a similarly metal-poor object HE 0507-1653 (Aoki et al. 2007). Christlieb et al. (2002, 2004) have also reported on a higher carbon isotopic ratio $\left({ }^{12} \mathrm{C} /{ }^{13} \mathrm{C}>30\right)$ for the star HE 0107-5240. Carbon abundance is found to be enhanced in stars that are formed from interstellar medium which is polluted by faint supernovae or spin stars (Umeda \& Nomoto 2005, Meynet et al. 2006, Chiappini 2013). The enhanced carbon can also be explained based on the mass transfer from a binary companion (McClure 1983, 1984). The binarity of HE $0110-0406$ is not yet confirmed. The enhanced ${ }^{12} \mathrm{C} /{ }^{13} \mathrm{C}$ ratio of a CEMP or $\mathrm{CH}$ star is an indicator of the mixing that had occurred in the progenitors. In the case of spin stars, the internal mixing is due to the convection driven by rapid rotation (Meynet et al. 2006, Hirschi 2007, Maeder et al. 2015) in which carbon is transported from the core into the shell where the CNO burning occurs. In AGB stars, the internal mixing caused by convection mixes the carbon from the surface into the $\mathrm{H}$ burning shell. In these processes ${ }^{12} \mathrm{C}$ is converted into ${ }^{13} \mathrm{C}$. Maeder et al. (2014) suggest that higher ${ }^{12} \mathrm{C} /{ }^{13} \mathrm{C}$ ratio may be a signature of the partial hydrogen burning in the AGB or spin stars.

CEMP stars exhibit a bimodal distribution of carbon with a plateau at $\mathrm{A}(\mathrm{C})=8.25$ for stars with $[\mathrm{Fe} / \mathrm{H}] \geq-3.4$ and a second plateau around $\mathrm{A}(\mathrm{C}) \sim 6.5$ for stars with $[\mathrm{Fe} / \mathrm{H}] \leq-3.4$ (Spite et al. 2013). Bonifacio et al. (2015) also confirms the bimodal distribution and suggest that the stars that fall in the high carbon band are the members of binary systems; the observed over-abundance of carbon is attributed to binary mass transfer. The stars in the lower band are mostly single and the observed carbon abundance is believed to be intrinsic. It reflects the abundance of carbon in the gas 
Table 3: Elemental abundances in HE 0110-0406

\begin{tabular}{|c|c|c|c|c|c|}
\hline & $\mathrm{Z}$ & solar $\log \epsilon^{*}$ & $\log \epsilon$ & {$[\mathrm{X} / \mathrm{H}]$} & {$[\mathrm{X} / \mathrm{Fe}]$} \\
\hline $\mathrm{C}$ & 6 & 8.43 & 7.85(syn) & -0.58 & 0.73 \\
\hline $\mathrm{N}$ & 7 & 7.83 & 7.15 (syn) & -0.68 & 0.63 \\
\hline $\mathrm{Na} \mathrm{I}$ & 11 & 6.24 & $5.53(1)$ & -0.71 & 0.60 \\
\hline $\mathrm{Mg} \mathrm{I}$ & 12 & 7.60 & $6.56(1)$ & -1.04 & 0.27 \\
\hline $\mathrm{Ca} \mathrm{I}$ & 20 & 6.34 & $5.02 \pm 0.13(4)$ & -1.32 & -0.01 \\
\hline Sc II & 21 & 3.15 & 1.70(syn) & -1.45 & -0.14 \\
\hline Ti I & 22 & 4.95 & $3.76 \pm 0.14(4)$ & -1.19 & 0.12 \\
\hline V I & 23 & 3.93 & 2.17(syn) & -1.76 & -0.45 \\
\hline $\mathrm{Cr}$ I & 24 & 5.64 & $4.29 \pm 0.20(3)$ & -1.35 & -0.04 \\
\hline Mn I & 25 & 5.43 & 4.30(syn) & -1.13 & 0.18 \\
\hline $\mathrm{Fe} \mathrm{I}$ & 26 & 7.50 & $6.19 \pm 0.09(27)$ & -1.31 & - \\
\hline $\mathrm{Fe}$ II & 26 & 7.50 & $6.21 \pm 0.12(2)$ & -1.29 & - \\
\hline Co I & 27 & 4.99 & $3.73 \pm 0.04(3)$ & -1.26 & 0.05 \\
\hline Ni I & 28 & 6.22 & $5.36(1)$ & -0.86 & 0.45 \\
\hline $\mathrm{Zn} \mathrm{I}$ & 30 & 4.56 & $2.84(1)$ & -1.72 & -0.41 \\
\hline Sr I & 38 & 2.87 & 2.34 (syn) & -0.53 & 0.78 \\
\hline Y II & 39 & 2.21 & $2.20(1)$ & -0.01 & 1.28 \\
\hline Ba II & 56 & 2.18 & $2.50($ syn $)$ & 0.32 & 1.61 \\
\hline La II & 57 & 1.10 & $1.07(1)$ & -0.03 & 1.26 \\
\hline Ce II & 58 & 1.58 & $1.49 \pm 0.21(3)$ & -0.09 & 1.20 \\
\hline Pr II & 59 & 0.72 & $0.82 \pm 0.09(2)$ & 0.10 & 1.39 \\
\hline Nd II & 60 & 1.42 & $1.49 \pm 0.18(4)$ & 0.07 & 1.36 \\
\hline Sm II & 62 & 0.96 & $0.90 \pm 0.14(4)$ & -0.06 & 1.23 \\
\hline $\mathrm{Eu}$ II & 63 & 0.52 & $-0.25(1)$ & -0.77 & 0.52 \\
\hline
\end{tabular}

* Asplund (2009); The number inside the parenthesis indicates the number of lines used for the abundance determination.

cloud from which it is formed. The location of HE 0110-0406, in the $\mathrm{A}(\mathrm{C})$ vs $[\mathrm{Fe} / \mathrm{H}]$ diagram (Figure 3 ) indicates that the carbon enhancement in HE 0110-0406 may be extrinsic.

The HE 0110-0406 shows moderate enhancement of $\mathrm{Na}$ and slightly enhanced $\mathrm{Mg}$. The iron group elements such as $\mathrm{Cr}$ and $\mathrm{Co}$ are found to be near solar as seen in similar metal-poor stars. According to Turatto et al. (1998), such abundance patterns can be produced by faint supernovae. Iron group elements are produced in the deep interior of these objects. As they do not have sufficient energy to throw all the materials, only the outer layer containing the lighter elements are ejected and the heavy elements would fall back into the core. Thus, the star that born from the material swept by supernova remnants can show enhanced $\mathrm{Na}, \mathrm{Mg}$ and other lighter elements compared to the Fe group elements.

The analysis of light and heavy neutron-capture elements in HE 0110-0406 show that the star is more enriched in heavy elements compared to the light neutron-capture elements with a ratio [hs/ls] $\approx 0.33$ (where hs refers to $\mathrm{Ba}, \mathrm{La}, \mathrm{Ce}$ and $\mathrm{Nd}$, and ls refers to $\mathrm{Sr}$ and $\mathrm{Y}$ ). Yttrium and Zirconium are the most abundant products of the neutron-capture process in AGB models with metallicities higher than solar (Busso et al. 2001). These elements can be produced at large amounts even at moderate neutron exposures. $\mathrm{Ba}$, La, $\mathrm{Ce}$ and $\mathrm{Nd}$ are the dominant products in low metallicity $([\mathrm{Fe} / \mathrm{H}] \approx-1$ ) AGB stars. At lower metallicities, the availability of iron seed nuclei will be less in numbers and the neutron exposure increases with decrease in the number of seed nuclei. Hence, the first peak s-process elements are bypassed by the second and third peak elements that lead to the formation of 


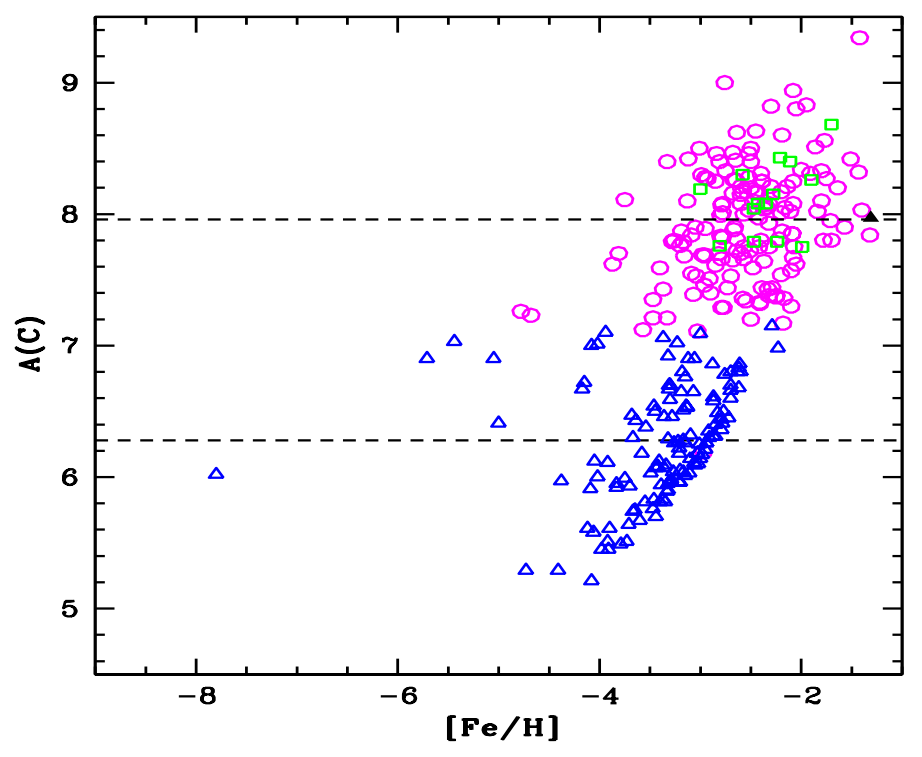

Figure 3: $\mathrm{A}(\mathrm{C})$ (corrected $\log \epsilon(\mathrm{C})$ ) vs $[\mathrm{Fe} / \mathrm{H}]$ diagram for CEMP stars from Yoon et al. (2016). CEMP-s stars and CEMP-no stars are represented by open circle (magenta) and open triangle (blue) respectively. CEMP-r/s stars are shown in open square (green). In this figure, HE 0110-0406 is represented by filled triangle (black). $\mathrm{A}(\mathrm{C})$ for HE 0110-0406 is estimated using the public online tool (Placco et al. 2014; http/://vplacco.pythonanywhere.com/). Dashed lines correspond to $\mathrm{A}(\mathrm{C})=$ 7.96 and 6.28 .

heavy s-process elements such as lead at low metallicity stars. [hs/ls] $<0$ is expected in AGB stars with metallicities higher than $[\mathrm{Fe} / \mathrm{H}]=-0.20$ where light s-process elements $\mathrm{Sr}, \mathrm{Y}$ and $\mathrm{Zr}$ are the dominant products (Goriely \& Mowlavi, 2000). According to Busso et al. (2001), AGB stars with metallicity $[\mathrm{Fe} / \mathrm{H}] \sim-1$ can have maximum of $[\mathrm{hs} / \mathrm{ls}]=1.2$. Hence a trend of increasing [hs/ls] with decreasing metallicity is expected. Mass as well as the number of thermal pulses can also affect the $[\mathrm{hs} / \mathrm{ls}]$ ratio. As most of the CEMP stars are found to be in binary systems, their observed abundance patterns may reflect the abundance in their AGB progenitors. Despite of certain amount of dilutions, the observed abundance of light as well as heavy s-process elements in CEMP stars are the implications of neutron-capture efficiency in the AGB stars from which they have accreted the material.

\section{Conclusion}

The object HE 0110-0406 was identified as a potential CH star candidate by Goswami (2005). It was identified from the low-resolution spectroscopic analysis of a sample of high latitude faint carbon stars in Chrislieb et al. (2001). We have performed a detailed chemical composition study for this object and confirmed its classification. The analysis is based on a high resolution spectrum obtained using HCT/HESP. The object with a metallicity $\sim-1.30$, and carbon enhancement with ([C/Fe] $\approx 0.73$ ) exhibits spectral features that are characteristics of $\mathrm{CH}$ stars. Kinematic analysis of $\mathrm{HE}$ 0110-0406, shows this object to be a thin disk object with a 94\% probability. Light elements as well as Fe-peak elements show abundance patterns as seen in similar metal-poor stars and neutron-capture elements show significant enhancement. The object is more enhanced in heavy s-process elements compared to the lighter s-process elements. The estimated [hs/ls] ratio is found to be around 0.15 . While the abundance patterns of light and Fe-peak elements may be due to the origin of the star from 
an interstellar medium enriched by the remnants of faint supernovae, the observed enhancement of neutron-capture elements require a binary mass transfer scenario. However, the binarity of this object is not yet confirmed. A systematic radial velocity monitoring of the object may help to confirm the binary nature of the object.

\section{Acknowledgement}

M.P. is a JRF under the DST project SERB project No. EMR/2016/005283; funds from this project is gratefully acknowledged. AG would like to acknowledge the financial support from BINA projects DST/INT/Belg/P-02 (India) and BL/11/IN07 (Belgium).

\section{References}

Aoki W., Beers T. C., Christlieb N. et al. 2007, ApJ, 655, 492

Arentsen A., Starkenburg E., Shetrone M. D. 2019, A\&A, 621, A108

Asplund M., Grevesse N., Sauval A. J. 2009, ARA\&A, 47, 481

Barbuy B., Cayrel R., Spite M. et al. 1997, A\&A, 317, L63

Beers T. C. 1992, AJ, 103, 1987

Beers T. C., Christlieb N. 2005, ARA\&A, 43, 531

Bidelman W. P. 1956, Vist. Astron., 2, 1428

Bonifacio P., Caffau E., Spite M. et al. 2015, A\&A, 579, A28

Busso M., Gallino R., Lambert D. L. et al. 2001, ApJ, 557, 802

Chiappini C. 2013, Astronomische Nachrichten, 334, 595

Christlieb N. 2003, Rev. Mod. Astron., 16, 191

Christlieb N., Bessell M. S., Beers T. C. et al. 2002, Natur, 419, 904

Christlieb N., Gustafsson B., Korn A. J. et al. 2004, ApJ, 603, 708

Goriely S., Mowlavi N. 2000, A\&A, 362, 599

Goswami A. 2005, MNRAS, 359, 531

Goswami A., Bama P., Shantikumar N. S. et al. 2007, MNRAS, 35, 339

Goswami A., Karinkuzhi D., Shantikumar N. S. 2010, MNRAS, 402, 111

Hansen T. T., Andersen J., Nordström B. et al. 2016a, A\&A, 586, A160

Hansen T. T., Andersen J., Nordström B. et al. 2016b, A\&A, 588, A3

Hirschi R. 2007, A\&A, 461, 571

Lucatello S., Tsangarides S., Beers T. C. et al. 2005, ApJ, 625, 825

Maeder A., Meynet G., Chiappini C. 2015, 576, A56

Masseron T., Johnson J. A., Plez B. et al. 2010, A\&A, 509, 93

McClure R. D. 1983, ApJ, 208, 264

McClure R. D. 1984, ApJ, 280, 31

McWilliiam A. 1998, AJ, 115, 1640

Meynet G., Ekström S., Maeder A. 2006, A\&A, 447, 623

Norris J. E., Ryan S. G., Beers T. C. 1997, ApJ, 488, 350

Placco V. M., Frebel A., Beers T. C. et al. 2014, ApJ, 797, 21

Preston G. W., Sneden C. 2001, AJ, 122, 1545

Prochaska J. X., McWilliam A. 2000, ApJ, 537, 57

Secchi A. 1868, Sugli spettri prismatici delle stelle fisse (Roma: Trip. Belle Arti), 11

Sneden C. 1973, PhD thesis, Univ. Texas

Sneden C., McWilliam A., Preston G. W. et al. 1996, ApJ, 467, 819

Spite M., Caffau E., Bonifacio P. et al. 2013, A\&A, 552, A107

Turatto, M., Mazzali P. A., Young T. R. et al. 1998, ApJ, 498, L129

Umeda H., Nomoto K. 2005, ApJ, 619, 427

Yoon J., Beers T. C., Placco V. M. et al. 2016, ApJ, 833, 20 